\title{
cc.eative
}

ISSN 1855-3966 (printed edn.), ISSN 1855-3974 (electronic edn.)

\author{
ARS MATHEMATICA CONTEMPORANEA 16 (2019) 157-171 \\ https://doi.org/10.26493/1855-3974.997.7ef \\ (Also available at http://amc-journal.eu)
}

\section{Regular polygonal systems}

\author{
Jurij Kovič * \\ Institute for Mathematics, Physics and Mechanics, Jadranska 19, 1000 Ljubljana and \\ FAMNIT, University of Primorska, Glagoljaška 8, 6000 Koper, Slovenia
}

Received 19 December 2015, accepted 4 March 2018, published online 18 November 2018

\begin{abstract}
Let $M=M(\Omega)$ be any triangle-free tiling of a planar polygonal region $\Omega$ with regular polygons. We prove that its face vector $f(M)=\left(f_{3}, f_{4}, f_{5}, \ldots\right)$, its symmetry group $S(M)$ and the tiling $M$ itself are uniquely determined by its boundary angles code $c_{a}(M)=c_{a}(\Omega)=\left(t_{1}, \ldots, t_{r}\right)$, a cyclical sequence of numbers $t_{i}$ describing the shape of $\Omega$.
\end{abstract}

Keywords: Regular polygonal system, boundary code, face vector, symmetry group, reconstructibility from the boundary.

Math. Subj. Class.: 05B40, 05B45

\section{Introduction}

Systems of regular (planar or spherical) polygons joined edge to edge arise in various contexts (in tilings, in polyhedral maps, in nature, in chemistry, in art). A rich theory of such systems may be developed. Researchers usually focus on some particular class of such systems (defined by some conditions), try to determine all its elements and explore various questions related to their combinatorial description, parameters, enumeration, characterization, classification, coding, etc. To unify the investigations of such objects and to emphasize their common characteristics we propose a general concept of a regular polygonal system and make some first few steps towards a general theory of such systems.

Here we give definitions, examples and remarks; the general reconstruction problem is presented in Section 2; results are gathered in Section 3.

A polygonal system $M$ is a (finite or infinite) incidence structure $M=(V, E, F)$ whose elements are called vertices, edges and faces: faces are abstract polygons - cyclical sequences of vertices $\left(v_{1}, \ldots, v_{n}\right)$, and edges are pairs of vertices $\left\{v_{i}, v_{i+1}\right\}$. Two faces are

* This work is supported in part by the Slovenian Research Agency (research program P1-0294 and research project N1-0032).

E-mail address: jurij.kovic@siol.net (Jurij Kovič)

(ㄷ)(7) This work is licensed under https://creativecommons.org/licenses/by/4.0/ 
incident if they share an edge, two edges are incident if they share a vertex. If $M$ consists only of $n$-gons it is called a $n$-system; if the number of these $n$-gons is $m$, it is a $n_{m}$-system. If all these faces are congruent polygons $X$, it is a monohedral system denoted $X_{m}$. If $M$ consists only of $n_{1}$-gons, $n_{2}$-gons, $\ldots, n_{k}$-gons, it is called a $\left(n_{1}, \ldots, n_{k}\right)$ system. The face vector (or just the $f$-vector) of the polygonal system $M$ is the sequence $f(M)=\left(f_{3}, f_{4}, f_{5}, \ldots\right)$ where $f(i)=f_{i}(M)$ are the numbers of its faces with $i$ edges. We use also the notation $f(M)=\left(3_{f(3)}, 4_{f(4)}, 5_{f(5)}, \ldots\right)$.

A planar or spherical polygon $P$ is called regular if there is a cyclic group $G=\langle R|$ $\left.R^{n}=I\right\rangle$ of rotations acting transitively on the vertices and edges of $P$, and if its boundary $\partial P$ is simply connected (thus we exclude star polygons, as in Kepler solids). A regular polygonal system $M=M(\Omega)$ consists of regular polygons joined edge to edge, covering a polygonal planar or spherical region $\Omega$. The symmetry group of $M=M(\Omega)$ is the group of the rotations and reflections of Euclidean space $E^{2}$ or $E^{3}$ preserving the incidences in $M$.

A code $c(X)$ of a given mathematical object $X$ is any (not necessarily discrete) structure from which $X$ can be completely reconstructed (up to isomorphism). A boundary code of a given $m$-dimensional object $X$ is any code $c(\partial X)$ of the shape of its $(m-1)$ dimensional boundary $\partial X$. The boundary angles code of the planar or spherical regular polygonal system $M=M(\Omega)$ covering (tiling) a polygonal region $\Omega$ is the cyclical sequence $c_{a}(M)=c_{a}(\Omega)=\left(t_{1}, t_{2}, \ldots, t_{r}\right)$ of the angles $t_{i}=180^{\circ}-\alpha_{i} \in\left(-180^{\circ}, 180^{\circ}\right)$, where $\alpha_{i}$ are the interior angles between adjacent edges $A_{i-1} A_{i}$ and $A_{i} A_{i+1}$ of $\Omega$. The boundary faces-edges code $c_{f, e}(M)$ is the cyclical sequence of symbols $f(i)_{e(i)}$, where $f(i)$ is the number of edges of $i$-th boundary face and $e(i)$ is the number of the boundary edges of this face (see examples in Appendix A). A few examples and remarks will help us better understand these definitions and the motivation for them.

In chemistry, the mathematical model of benzenoid molecules are called benzenoid systems or polyhexes (composed of regular hexagons). Similar systems are polydiamonds (composed of equilateral triangles) and polyominoes (composed of squares) (see Figure 1).

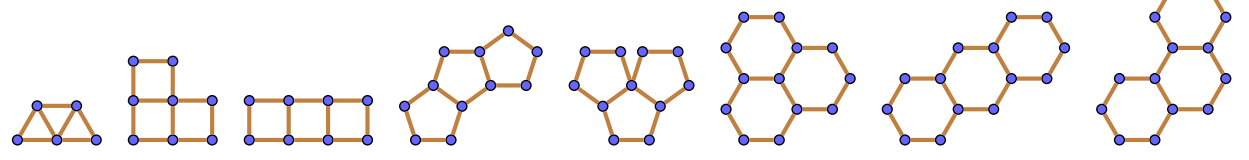

Figure 1: Planar regular $n_{3}$-systems for $n=3,4,5,6$.

The motivation for the boundary angles code $c_{a}$ comes from the "turtle geometry" [1]. The definition of boundary faces-edges code $c_{f, e}$ is motivated by the boundary codes of various $n$-systems presented below.

Several boundary codes exist for the planar regular hexagonal systems $B$. One of them is the boundary edges code [6]. This code $c_{e}(B)=\left(k_{1}, k_{2}, \ldots, k_{r}\right)$ is a cyclical sequence counting the numbers $k_{i}$ of boundary edges in boundary hexagons; we travel around the boundary in the clockwise direction, starting at any hexagon, and having the interior of $B$ always at our right (see [2, 7]).

The code for the regular planar triangular systems (or 3-systems) $T$ may be defined exactly in the same way - as the boundary edges code $c_{e}(T)$ counting the number of boundary edges in boundary triangles of $T$. Thus, for the 3-system $T$ in Figure 1 its code is $c_{e}(T)=(2,1,2)$. 
The case of the planar square systems (or 4-systems) $S$ is trickier. To get the right numbers in $c_{e}(S)$ we must count also the "zeros" of boundary edges in boundary vertices adjacent to no boundary edge. We can use also a simple boundary vertices-faces code $c_{v, f}(S)$, a cyclical sequence counting for each boundary vertex how many squares $(1,2$ or 3 ) are incident with that vertex. For the first 4-system $S$ in Figure 1 we have $c_{e}(S)=$ $(2,3,0,3)$ and $c_{v, f}(S)=(1,2,1,1,3,1,1,2)$.

There is also a code for the regular pentagonal systems $P$ (described in [3]) that actually counts the numbers of vertices incident only with boundary edges. For the second 5 -system from Figure 1 this code is $(3,3,2)$.

One can easily imagine polygonal systems composed of regular polygons with different numbers of edges, and also non-planar generalizations of these concepts.

Example 1.1. The face vector of the planar regular $(3,4,5)$-system $M$ in Figure 2 is $f(M)=\left(3_{3}, 4_{1}, 5_{2}\right)=T_{3} S_{1} P_{2}$, since there are three triangles $T\left(f_{3}=3\right)$, one square $S\left(f_{4}=1\right)$ and two pentagons $P\left(f_{5}=2\right)$. Its symmetry group $S(M)$ is generated by one reflection (over the vertical axis).

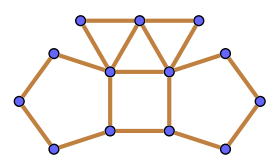

Figure 2: A polygonal system $M$ with the symmetry group $S(M)=\mathbb{Z}_{2}$.

Example 1.2. The boundary faces-edges code of the planar system $M$ in Figure 3 is $c_{f, e}(M)=\left(5_{4}, 4_{0}, 3_{2}, 4_{2}\right)$. The boundary angles codes $c_{a}(M)$ of the spherical systems with the same $c_{f, e}(M)$ depend on the size of the spherical triangle $T$. Increasing the size of $T$ the interior angles $\alpha_{i}$ increase as well, hence the angles $t_{i}=180^{\circ}-\alpha_{i}$ decrease. If $T$ is big enough, the angle between the pentagon and the triangle vanishes and we get a region with $c_{f, e}(M)=\left(5_{3}, 3_{1}, 4_{2}\right)$.

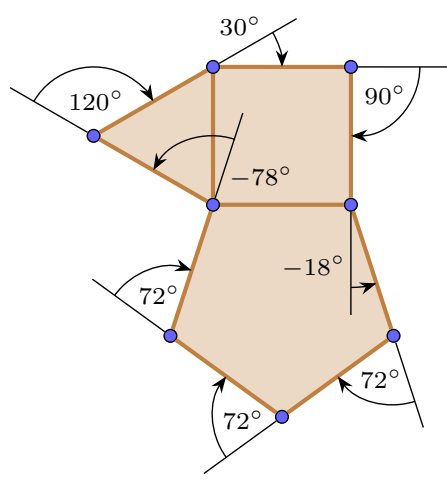

Figure 3: A planar regular polygonal $\left(3_{1}, 4_{1}, 5_{1}\right)$-system $M$ with the boundary angles code $c_{a}(M)=\left(120^{\circ}, 30^{\circ}, 90^{\circ},-18^{\circ}, 72^{\circ}, 72^{\circ}, 72^{\circ},-78^{\circ}\right)$.

The maps of Platonic solids are examples of regular 3-systems (tetrahedron $3_{4}$, octahedron $3_{8}$, icosahedron $3_{20}$ ), 4 -systems (cube $4_{6}$ ) and 5 -systems (dodecahedron $5_{12}$ ). The 
polygonal system of the square pyramid is $(B, C, D, E),(A, B, C),(A, C, D),(A, D, E)$, $(A, E, B)$, thus it is a $(3,4)$-system. The system $(A, B, C, D),(A, B, D, C)$ represents the Möbius band tiled with two quadrilaterals. In a polygonal system more than two faces may share the same edge, as in the "3-page book" $(A, B, C, D),(A, B, D, E),(A, B, F, G)$, which is a 3 -system, too.

\section{The reconstruction problem}

The first motivation for this research was the realization that it is possible to generalize the method that worked so well for benzenoid systems $B$ : "Encode the boundary of a system as a cyclical sequence of numbers, and then obtain various information about $B$ from its boundary code $b(B)$ " to other planar polycyclic molecules. Thus $b(B)$ was used to find various relations between the parameters of $B$, to determine its symmetry scheme, to calculate its face count, etc. (as in $[5,7,8]$ ). With this motivation in mind we have in the Introduction already defined two boundary codes $c_{e, f}(M)$ and $c_{a}(M)$. Now we can ask: Is it possible to reconstruct the face vector $f(M)$, the symmetry group $S(M)$ or even the whole planar regular polygonal system $M=M(\Omega)$ from its boundary code $c_{f, e}(M)$ or $c_{a}(M)$ ? Obviously, some regular polygonal systems $M$ are reconstructible from the chosen boundary code of the region $\Omega$ covered by $M$. How to characterize such systems? This question may be stated in a more general form: Given some class $\mathcal{C}$ of objects $X$ whose boundary is determined by some boundary code $c(\partial X)$ characterize those $X$ from $\mathcal{C}$ that can be reconstructed from its boundary code, in other words, find $X$ for which $c(\partial X)$ is also the code $c(X)$ of $X$.

The code is not necessarily a cyclical sequence of numbers. The boundary of any simply connected polycube $P$ (a solid composed of cubes of unit length joined face to face) may be coded with a graph $G(P)$ whose vertices are boundary square faces of $P$ and whose edges are labeled with angles $(0, \pi / 2,-\pi / 2)$ between adjacent boundary faces. The number of cubes in $P$, the symmetry group $S(P)$ and the polycube $P$ itself are obviously determined by its boundary. However, their actual reconstruction from the graph $G(P)$ is complicated.

The code is not always a discrete structure. In analysis, a differentiable function $f$ is reconstructible from its derivative $f^{\prime}$ by the Newton-Leibniz formula up to an additive constant $C$. Likewise, a harmonic function $f: \Omega \rightarrow \mathbb{R}$ is determined by its values on the boundary of $\Omega$.

Not all the codes of the same object contain the same amount of information. For example, two similar planar triangles have different codes (lengths $a, b, c$ and $a^{\prime}, b^{\prime}, c^{\prime}$ ), but the same boundary code (consisting of angles $\alpha, \beta, \gamma$ ). In some cases the chosen boundary code $c(\partial X)$ contains some additional information about the structure of $X$ that cannot be deduced from $\partial X$ alone. Thus it may happen that it is possible to reconstruct $X$ from the code of $\partial X$, although it is not possible to reconstruct $X$ only from $\partial X$.

Example 2.1. Let $\Omega$ be a planar polygonal region composed of a regular 12-gon and a square sharing one side. There are two tilings $M_{1}$ and $M_{2}$ of $\Omega$ with 12 squares $S$ and 20 equilateral triangles $T$, having the same boundary angles code $\left(90^{\circ}, 90^{\circ}, 60^{\circ}, 30^{\circ}, 30^{\circ}, 0^{\circ}\right.$, $30^{\circ}, 30^{\circ}, 30^{\circ}, 0^{\circ}, 30^{\circ}, 30^{\circ}, 30^{\circ}, 0^{\circ}, 30^{\circ}, 30^{\circ}, 30^{\circ},-90^{\circ}$ ), but different boundary faces-ed- 
ges codes (see Figure 4):

$$
\begin{aligned}
& c_{e, f}\left(M_{1}\right)=\left(4_{3}, 4_{0}, 3_{1}, 4_{1}, 3_{1}, 3_{0}, 3_{1}, 4_{1}, 3_{1}, 4_{1}, 4_{1}, 3_{1}, 4_{1}, 3_{1}, 3_{0}, 3_{1}, 4_{1}, 3_{1}, 4_{1}, 4_{0}\right), \\
& c_{e, f}\left(M_{2}\right)=\left(4_{3}, 3_{0}, 4_{1}, 3_{1}, 4_{1}, 4_{1}, 3_{1}, 4_{1}, 3_{1}, 3_{0}, 3_{1}, 4_{1}, 3_{1}, 4_{1}, 4_{1}, 3_{1}, 4_{1}, 3_{1}, 3_{0}, 3_{0}\right) .
\end{aligned}
$$

Hence, it is not possible to reconstruct $M$ from the shape of the boundary $\partial \Omega$, encoded by $c_{a}(\Omega)$. However, $M$ is reconstructible from $c_{f, e}(M)$, since there are only two possible tilings $M_{1}$ and $M_{2}$ of $\Omega$. In this sense, $c_{f, e}(M)$ is a stronger code (containing more information), and $c_{a}(M)=c_{a}(\Omega)$ is weaker (containing less information).

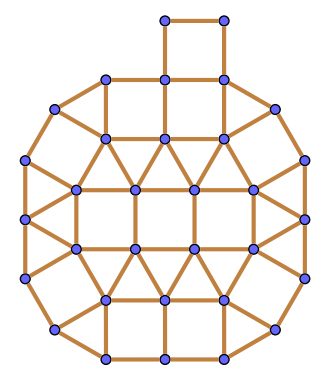

(a) $M_{1}$

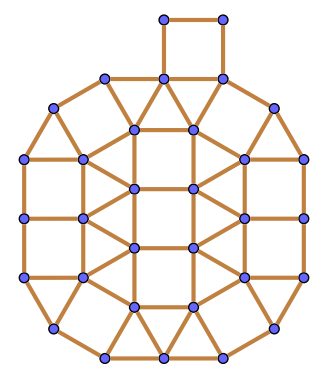

(b) $M_{2}$

Figure 4: Two different planar $(3,4)$-systems $M_{1}$ and $M_{2}$ with the same boundary and with the same face vector $\left(3_{20}, 4_{12}\right)$.

Remark 2.2. Two closed planar polygonal regions $\Omega$ and $\Omega^{\prime}$ may have the same boundary angles code $c_{a}=\left(t_{1}, \ldots, t_{r}\right)$, but if the ratio of the lengths of the corresponding edges $A_{i} A_{i+1}$ and $A_{i}^{\prime} A_{i+1}^{\prime}$ are not all the same, then the shapes of these regions are different. Note that the lengths of the edges of a region $\Omega$ tiled by regular polygons joined edge to edge are the same. This is the reason why the boundary angles code $c_{a}(\Omega)$ suffices to describe the shape of the boundary of $\Omega$, tiled by regular polygons. ${ }^{1}$

\section{Results}

In this Section we show: if $M$ is a regular triangle-free tiling of a planar polygonal region $\Omega$, then we can use $c_{a}(M)$ to find the face vector $f(M)$ (Theorems 3.1 and 3.8), to determine the symmetry group $S(M)$ (Theorem 3.12), or even to completely reconstruct $M=M(\Omega)$ (Theorem 3.5).

Theorem 3.1. Let $M=M(\Omega)$ be a planar regular $(3,4)$-system covering the polygonal region $\Omega$. Then the boundary angles code $c_{a}(M)=c_{a}(\Omega)$ determines the face vector $f(M)$ of $M$.

Proof. The area of $\Omega$ can be calculated from the boundary angles code $c_{a}(\Omega)=\left(t_{1}, \ldots, t_{n}\right)$ as follows: fix the coordinates of two adjacent vertices $A_{1}=(0,0)$ and $A_{2}=(1,0)$ of $\Omega$, use vectors to find the coordinates of other vertices $A_{i}$, triangulate $\Omega$ and sum the areas

\footnotetext{
${ }^{1}$ However, as we see in Figure 4, the tiling $M(\Omega)$ may not be uniquely determined by the shape of its boundary, although it is tiled by regular polygons.
} 
of all these triangles. The area of the regular $n$-gon $P_{n}$ with side 1 is $\operatorname{Area}\left(P_{n}\right)=n$. $\cot \left(360^{\circ} / n\right)$. These numbers are incommensurable at least for $n=3,4$ since $\operatorname{Area}\left(P_{3}\right)=$ $\sqrt{3} / 4$, Area $\left(P_{4}\right)=1$. Now it is easy to see that the integer solutions of the equation $x_{1}(\sqrt{3}) / 4+y_{1}=x_{2}(\sqrt{3}) / 4+y_{2}$ are possible only if $x_{1}=x_{2}, y_{1}=y_{2}$. Thus $f(M)$ is determined by $c_{a}(M)$. Solving the equation $x_{1}(\sqrt{3}) / 4+y_{1}=\operatorname{Area}(M)$ is easy, since the calculated expression for $\operatorname{Area}(M)$ must appear in this form, from which we just read $x_{1}$ and $y_{1}$.

Theorem 3.2. The sum of the angles in the boundary angles code $c_{a}(\Omega)=\left(t_{1}, t_{2}, \ldots, t_{r}\right)$ of a planar polygonal region $\Omega$ is $t_{1}+t_{2}+\cdots+t_{r}=360^{\circ}$.

Proof. This follows from the formula $\sum_{i=1}^{n} \alpha_{i}=(n-2) \cdot 180^{\circ}$ for the sum of the interior angles of a $n$-gon: $\sum_{i=1}^{n} t_{i}=\sum_{i=1}^{n}\left(180^{\circ}-\alpha_{i}\right)$. Another proof in the context of the "turtle geometry" is given in [1, p. 175].

Regular (planar or spherical) $n$-gons are usually denoted by the symbol $\{n\}$ or just $n$. The vertex type of the interior vertex of the regular polygonal system $M$ is defined as the cyclical sequence $(a . b . c . .$.$) ) of the faces a, b, c, \ldots$ surrounding it. The planar vertex types in this notation are listed in [4]. It is easy to check the following very useful observation ([4, p. 60]).

Theorem 3.3. If the planar regular $n_{1}$-gon, $\ldots, n_{r}$-gon surround a vertex without gaps and overlaps, then $3 \leq r \leq 6$ and $\left(n_{1}-2\right) / n_{1}+\cdots+\left(n_{r}-2\right) / n_{r}=2$, hence there are 21 types of vertices surrounded by regular polygons in a plane without gaps or overlaps:

$\begin{array}{ccccccc}3.3 .3 .3 .3 .3, & 3.3 .3 .6, & 3.3 .3 .4 .4, & 3.3 .4 .3 .4, & 3.3 .4 .12, & 3.4 .3 .12, & 3.3 .6 .6, \\ 3.6 .3 .6, & 3.4 .4 .6, & 3.4 .6 .4, & 3.7 .42, & 3.9 .18, & 3.8 .24, & 3.10 .15, \\ 3.12 .12, & 4.4 .4 .4, & 4.5 .20, & 4.6 .12, & 4.8 .8, & 5.5 .10, & 6.6 .6 .\end{array}$

Theorem 3.4. The possible (interior or boundary) vertex types in spherical regular polygonal systems are (if we exclude spherical 2-gons):

- 5 triangles: 3.3.3.3.3;

- 4 triangles: 3.3.3.3.4, 3.3.3.3.5;

- 3 triangles: $3.3 .3,3.3 .3 .4,3.3 .3 .5$;

- 2 triangles: $3.3,3.3 . m, 3 . m .3(m \geq 4), 3.3 .4 .5,3.4 .3 .5,3.3 .5 .5$;

- 1 triangle: 3 , 3.m ( $m \geq 4)$, 3.4.n, 3.n.4 ( $n \geq 4)$, 3.5.n, 3.n.5 ( $n \geq 5)$;

- 0 triangles: $m, 4 . m, 4.4 . m, 4 . m .4$ ( $m \geq 4)$, 4.5.n, $4 . n .5$ (19 $\geq n \geq 4)$, 5.5.5.

Proof. We just use the fact that for the interior angle $\alpha_{n}$ of the spherical $n$-gon it holds that $180^{\circ}>\alpha_{n}>180^{\circ}-360^{\circ} / n$, and check all the possible cases. A similar treatment of faces is given in Appendix B.

Theorem 3.5. Let $M=M(\Omega)$ be a planar regular polygonal system covering the polygonal region $\Omega$. If $M$ contains no triangles then it is reconstructible from its boundary angles code $c_{a}(M)$. Likewise, $M$ is reconstructible from $c_{a}(M)$ also in the case $M$ is without squares and hexagons. 
Proof. The theorem is certainly true if $M$ contains only one face. Now suppose it is true for the systems with $m$ or less faces and let $M=M(\Omega)$ be a system with $m+1$ faces. Since the sum of the $r$ inner angles $\alpha_{i}$ of $\Omega$ is $\sum_{i=1}^{r} \alpha_{i}=(r-2) \cdot 180^{\circ}$, at least one $\alpha_{i}$ must be smaller than $180^{\circ}$. The vertex $A_{i}$ of such $\alpha_{i}<180^{\circ}$ is incident with at most three regular polygons: with two or three triangles, with one triangle and one square or pentagon or with two squares, or with a single $n$-gon with the inner angle $(n-2) \cdot 180^{\circ} / n$.

Hence, if $M$ is triangle-free, then the angle $\alpha_{i}<180^{\circ}$ is incident with only one $n$-gon $P_{n}$ whose interior angle is $(n-2) \cdot 180^{\circ} / n=\alpha_{i}$. Removing this $P_{n}$ from $M$ we get a smaller system, whose tiling is unique, by the induction hypothesis. Hence the position of each polygon in $M$ is uniquely determined.

Similarly, if there are no squares and hexagons in $M$, then every interior angle $\alpha_{i}<$ $180^{\circ}$ is incident either with a single polygon $P_{n}$ (and then we proceed as before) or with a triangle and a pentagon. But there is no planar interior vertex type containing faces 3 and 5 (see Theorem 3.3). Therefore both the vertices of the edge $A C$ shared by a boundary triangle $A B C$ and a pentagon are boundary vertices (see Figure 5). If we interchange the
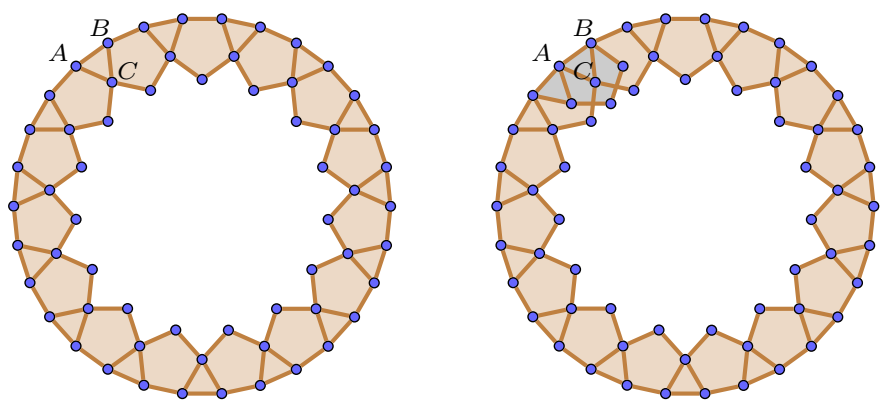

Figure 5: Left: A $(3,5)$-system with two boundary components and with the cyclical symmetry group $C_{15}$. Right: An illustration of the fact that such system cannot have two different tilings.

positions of two adjacent boundary faces 3 and 5 along the adjacent boundary edges then the interchanged face 5 covers a neighbourhood of $C$ (as in Figure 5 right). So there is only one possible tiling of $M$ in the neighbourhood of all boundary points incident with 3.5 or 5.3. Removing this triangle and pentagon we get a smaller region for which the theorem is true by the induction hypothesis.

Remark 3.6. It may happen that the boundary of $M-P_{n}$ is no longer a simple closed curve (see Figure 6); it may have crossings and it may have more than one component. However, by repeating the process of removing the polygons corresponding to interior angles $\alpha_{i}<180^{\circ}$ from the system and calculating the boundary angles code of the smaller system we may find the exact locations of each face in the system algorithmically. For example, removing a boundary square $P_{4}$ from $M$ changes the boundary code from $c_{a}(M)=$ $\left(t_{1}, t_{2}, \ldots, t_{i-1}, t_{i}=\alpha_{4}=90^{\circ}, t_{i+1}, \ldots, t_{r}\right)$ into $c_{a}\left(M-P_{4}\right)=\left(t_{1}, t_{2}, t_{i-1}+\alpha_{4}, \alpha_{4}-\right.$ $\left.180^{\circ}, \alpha_{4}+t_{i+1}, \ldots, t_{r}\right)$, as in Figure 6. For $n \geq 5$ we get a more complicated formula for $c_{a}\left(M-P_{n}\right)$, dependent on how many successive angles $t_{i}, t_{i+1}, \ldots t_{i+k}$ in $c_{a}(M)$ are equal to $(n-2) \cdot 180^{\circ} / n$.

Just as in the planar case, there are polygonal regions on the sphere admitting more 

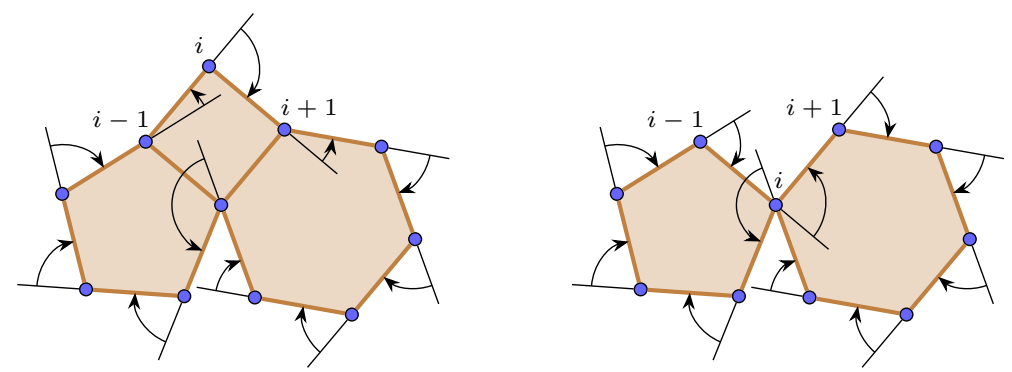

Figure 6: Removing of a boundary face may produce a system that is no more faceconnected.

than one regular tiling, too. A spherical pentagon, covered by five regular triangles sharing a vertex of the spherical icosahedron is an example of such a region.

Theorem 3.7. Let $M$ be a spherical regular polygonal system $M$ without triangles and squares. If the boundary of $M$ has only one component, then $M$ is reconstructible from its boundary faces-edges code $c_{f, e}(M)$.

Proof. By Theorem 3.4 $M$ has no interior points. Hence there can be no cycle composed of adjacent faces in $M$, and $c_{f, e}(M)$ completely describes the system $M$.

Theorem 3.8. The face vector $f(M)=\left(f_{3}, f_{4}, f_{5}, \ldots\right)$ and consequently also the number offaces $f=f_{3}+f_{4}+f_{5}+\cdots$ of any regular planar polygonal system $M=M(\Omega)$ without hexagons or triangles and with all sides of length 1 is uniquely determined by the area of $\Omega$, and this area is uniquely determined by its boundary angles code $c_{a}(\Omega)=c_{a}(M)$.

Proof. If $M=M(\Omega)$ has at most two faces, then its face vector is obviously determined by its boundary code, except in the case of a hexagon, which may be decomposed into six triangles. Suppose the theorem is true for any system with $n$ faces. Take a system $M$ with $n+2$ faces. In every boundary vertex with a positive angle $t_{i}$ we can repeat the procedure of taking out the polygons of $M$ as in the proof of Theorem 3.5. In the boundary vertices with the interior angles filled with $3.4,4.3,3.5,5.3$, we take away from $M$ both combinations and get two smaller systems $M^{*}$ and $M^{* *}$ composed of $n$ faces which must have the same area, hence they have the same face vector by the induction hypothesis. Therefore $M$ has the unique face vector, too.

Theorem 3.9. The symmetry group $S(M)$ of a planar or spherical regular polygonal system $M=M(\Omega)$ is a subgroup of $S(\Omega)=S(\partial \Omega)$.

Proof. Any rotation or reflection preserving $M$ preserves the region $\Omega$, tiled by the polygons of $M$. The symmetry group of the boundary of $\Omega$ is obviously isomorphic to the symmetry group of $\Omega$.

A symmetry of the boundary of $\Omega$ does not necessarily induce a symmetry of $M=$ $M(\Omega)$. For example, if we glue together the two 12-gons from Figure 4 (without the added top square) along the vertical edge, we get a region with two reflection symmetries (over the vertical axis and over the horizontal axis), yet its tiling has only one reflection symmetry (over the horizontal axis). 
Theorem 3.10. If there is only one regular polygonal system $M=M(\Omega)$ covering the region $\Omega$ then the groups $S(M)$ and $S(\Omega)$ are isomorphic.

Proof. If the tiling $M$ of $\Omega$ with regular polygons is unique, then every symmetry of $\Omega$ automatically induces an automorphism of $M$.

Lemma 3.11. Let $\Omega$ be a planar polygonal system with given lengths of its edges $l_{i}=$ $A_{i} A_{i+1}$ and with the boundary angles code $c(\Omega)=\left(t_{1}, \ldots, t_{r}\right)$.

(i) If $t_{i+k}=t_{k}$ and if $l_{i+k}=l_{k}$ for some $k \geq 2$, then $\Omega$ has a rotational symmetry for the angle $360^{\circ} /(r / k)$.

(ii) If $c_{a}(\Omega)=\left(t_{1}, \ldots, t_{r}\right)=\left(t_{r}, \ldots, t_{1}\right)$ and if the cyclical sequence $c_{l}=\left(l_{1}, l_{2}, \ldots\right.$, $\left.l_{r}\right)$ of the lengths $l_{i}$ of the boundary edges $A_{i} A_{i+1}$ has a reflection symmetry, then $\Omega$ has a reflection symmetry.

Proof. (i) This is obviously true for $k=1$, for in that case we have $c_{a}(\Omega)=(t, t, \ldots, t)$, hence all $t_{i}$ are the same, and all $l_{i}$ are the same, hence $\Omega$ is a regular polygon $P_{r}$ invariant for the rotation for the angle $360^{\circ} / r$.

In the case $k=2$ we have $c_{a}(\Omega)=(t, s, t, s, \ldots, t, s)$. Removing from $\Omega$ the congruent triangles $\triangle A_{1} A_{2} A_{3}, \triangle A_{3} A_{4} A_{5}, \ldots, \triangle A_{2 n-1} A_{2 n} A_{1}$ we get a region with $r / k=r / 2$ boundary edges where all $t_{i}$ are the same and all $l_{i}$ the same (case $k=1$ ) and for which we know (i) is true; hence $\Omega$ has the rotation for the angle $360^{\circ} /(r / 2)$, too.

Similarly, for the $k \geq 3$ we remove $r / k=n$ congruent triangles from $\Omega$ to get a smaller region with $r-\frac{r}{k}=n k-n=n(k-1)$ boundary angles and with a period $k-1$, hence by the induction hypothesis having a rotation $R_{n}$ where $n=\frac{r}{k}=\frac{n(k-1)}{k-1}$. Hence $\Omega$ has the rotation for the angle $360^{\circ} /(r / k)$, too.

(ii) Reflection symmetry of the sequence $c_{l}(\Omega)$ may have three forms ${ }^{2}$ :

(a) $c_{l}=(y, z, \ldots, b, a, a, b, \ldots, y, z)$,

(b) $c_{l}=(x, y, z, \ldots, b, a, a, b, \ldots, y, z)$,

(c) $c_{l}=(x, y, z, \ldots, b, a, a, b, \ldots, y, z, w)$,

and the same holds for the cyclical sequences of the lengths $l_{i}$.

Obviously (ii) is true if $\Omega$ has $3,4,5$ or 6 sides, since the only possible cases of $c_{l}(\Omega)$ are: $(x, y, y),(y, z, z, y),(x, y, y, z),(x, y, z, z, y),(x, y, z, z, y, w)$ and $(x, y, z, z, y, x)$. Now we can use a simple induction argument to see that if $\Omega$ has more sides than 6 , then we can cut it in two pieces with the boundary angles code of the types (c) or (b), and each of these two pieces has the same reflection symmetry (whose axis is the symmetral of the same line $X Y$ ), hence $\Omega$ has the same reflection symmetry (see Figure 7 middle). This is clear for regions of the type (a); the regions of the types (b) and (c) are obtained from a region of the type (a) by glueing one or two triangles to it.

Theorem 3.12. Let $M=M(\Omega)$ be a regular planar polygonal system without triangles covering the region $\Omega$. Then the symmetry group $S(M)$ of $M$ and the center of the rotation $R_{n}$ are determined by its boundary angles code $c_{a}(M)$ as follows:

(i) if $c_{a}(M)$ has a reflection symmetry $c_{a}(M)=\left(t_{1}, \ldots, t_{r}\right)=\left(t_{r}, \ldots, t_{1}\right)$ then $M$ has a reflection symmetry;

\footnotetext{
${ }^{2}$ The letters $x, y, z, a, b, \ldots$ are used here to denote lengths $l_{i}$ of boundary edges of $\Omega$.
} 

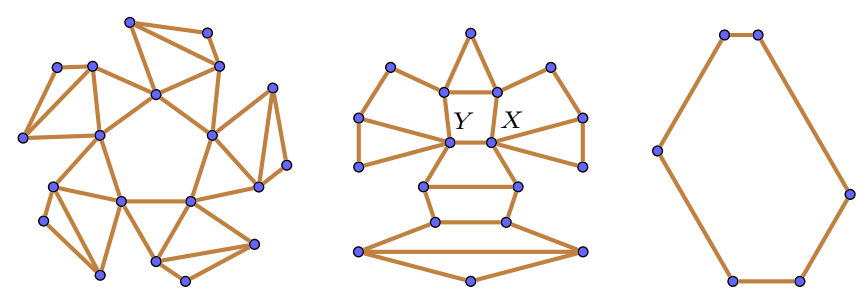

Figure 7: Left: a region with rotational symmetry; middle: a region with reflection symmetry; right: a region whose all boundary angles are identical (equal to $120^{\circ}$, as in a regular hexagon), yet it has no symmetry.

(ii) if $c_{a}(M)=\left(t_{1}, \ldots, t_{r}\right)$ is a periodical cyclical sequence: $t_{i+k} \equiv t_{i}$ for some $k \geqslant 2$ then $M$ has a rotational symmetry for the angle $360^{\circ} /(r / k)$;

(iii) if $f \equiv 1(\bmod n)$ then the center of the rotation $R_{n}$ is in a face;

(iv) if $f \equiv 0(\bmod n)$ and $n>2$ then the center of the rotation is in the vertex (if $n>2$ );

(v) if $f \equiv 0(\bmod n)$ and $n=2$ then the center of the rotation is in an edge.

Proof. By Theorem 3.5, $M=M(\Omega)$ is uniquely determined by its boundary angles code $c_{a}(M)=c_{a}(\Omega)$. By Theorem 3.9, the group of symmetries of $M$ is isomorphic to the group of symmetries of the boundary of $\Omega$. Now (i) and (ii) follow directly from Lemma 3.11. Indeed, if the boundary angles code $c_{a}(M)=c_{a}(\Omega)$ of length $r$ remains the same after the shift $k(\bmod m)$, then $r=k n$ is divisible by $k$ and $\Omega$ has a rotational symmetry $R_{n}$ (see Lemma 3.11).

Likewise, the reflection symmetry of $c_{a}(\Omega)$ implies the reflection symmetry of $\Omega$, since all the edges of $\Omega$ are of the same size (since they are tiled by the regular polygons of $M$ joined edge to edge) and we can apply Lemma 3.11. Now we use again $S(\Omega)=S(M)$, implied by Theorem 3.5.

The center of the rotational symmetry $R_{n}$ of any planar regular polygonal system can be in a vertex (this is possible only in the cases when $n=3,4,6$ ), in an edge center (this is possible only if $n=2$ ) or in a center of a $m$-gon, where $n$ divides $m$. If there are no triangles in the system, and if $n$ is odd, then the center of the rotation $R_{n}$ is in a face center.

If the rotational symmetry $R_{n}$ has the center in a $m$-gon, then $f_{m} \equiv 1(\bmod n)$ while the number of all other $i$-gons $f_{i}$ is divisible by $n$, hence $f \equiv 1(\bmod n)$. If the center of the rotation is in a vertex or in an edge center, then $f_{i} \equiv 0(\bmod n)$ for every $i$, hence $f \equiv 0(\bmod n)$. By Theorem 3.8 the face vector of $M$ without triangles may be obtained from the boundary angles code $c_{a}(M)$ so the numbers $f_{i}$ are known, hence we can easily distinguish between the two possible cases with rotational symmetry: $f \equiv 1(\bmod n)$ and $f \equiv 0(\bmod n)$.

If $f \equiv 0(\bmod n)$ and $n>2$ then the center of the rotation must be in a vertex. If $f \equiv 0(\bmod n)$ and $n=2$ then the center of the rotation must be in an edge.

Remark 3.13. So the information hidden in the boundary angles code suffices to find out whether the center of rotation is located in a face, in a vertex or in the middle of an edge. ${ }^{3}$

\footnotetext{
${ }^{3}$ Another possible approach to this question is to calculate the center of rotation directly via orbit barycenters, and compare its coordinates with the coordinates of all the vertices, face centers and edge centers of the tiling $M$.
} 


\section{Summary and open questions}

We proposed a concept of a regular polygonal system, a mathematical model of chemical (planar or non-planar) molecules composed of chains of atoms forming regular $n$-gonal cycles of equal or various length $(n=3,4,5,6, \ldots)$.

We proved that the structure (and hence the symmetry group) of any regular planar polygonal system $M$ without triangles or without squares and hexagons is reconstructible from its boundary angles code $c_{a}(M)$.

The proof of Theorem 3.1 implicitly uses a simple incommensurability argument. This type of argument can be generalized as in the following definition and lemma:

Definition 4.1. The quantities $q_{1}, q_{2}, \ldots, q_{n}$ are called incommensurable, if they satisfy the following condition: if two linear combinations of these quantities with integer coefficients are the same, i.e. $\sum_{i=1}^{n} a_{i} q_{i}=\sum_{i=1}^{n} b_{i} q_{i}$, then their corresponding coefficients must be the same, i.e. $a_{i}=b_{i}$.

From this definition immediately follows:

Lemma 4.2. If any quantity $q$ can be expressed as an integer linear combination

$$
q=\sum_{i=1}^{n} a_{i} q_{i}
$$

of incommensurable quantities, then the coefficients $a_{i}$ are uniquely determined by $q$.

Conjecture 4.3. The areas of all planar regular polygons with the same side length (except the triangle or hexagon) are incommensurable quantities.

Conjecture 4.4. The volumes of all Platonic and Archimedean solids with the same side are incommensurable quantities.

If one could prove Conjecture 4.3, this would automatically prove also Theorem 3.8. However, the converse is not true. If one could prove Conjecture 4.4 this would prove another conjecture:

Conjecture 4.5. If we glue together copies of Platonic and Archimedean solids (with unit sides) face to face then we can find the number of each of them just by knowing the volume of such composed solid.

\section{References}

[1] H. Abelson and A. diSessa, Turtle Geometry: The Computer as a Medium for Exploring Mathematics, MIT Press Series in Artificial Intelligence, MIT Press, Cambridge, Massachusetts, 1981.

[2] A. T. Balaban, Chemical graphs-vii: Proposed nomenclature of branched cata-condensed benzenoid polycyclic hydrocarbons, Tetrahedron 25 (1969), 2949-2956, doi:10.1016/ s0040-4020(01)82827-1.

[3] M. Deza, P. W. Fowler and V. P. Grishukhin, Allowed boundary sequences for fused polycyclic patches and related algorithmic problems, J. Chem. Inf. Comput. Sci. 41 (2001), 300-308, doi: 10.1021/ci000060o.

[4] B. Grünbaum and G. C. Shephard, Tilings and Patterns, W. H. Freeman and Company, New York, 1987. 
[5] I. Gutman and S. J. Cyvin, Introduction to the Theory of Benzenoid Hydrocarbons, SpringerVerlag, Berlin, 1989, doi:10.1007/978-3-642-87143-6.

[6] P. Hansen, C. Lebatteux and M. Zheng, The boundary-edges code for polyhexes, J. Mol. Struct. THEOCHEM 363 (1996), 237-247, doi:10.1016/0166-1280(95)04139-7.

[7] J. Kovič, How to obtain the number of hexagons in a benzenoid system from its boundary edges code, MATCH Commun. Math. Comput. Chem. 72 (2014), 27-38, http: / / match • pmf . kg . ac.rs/electronic_versions/Match72/n1/match72n1_27-38.pdf.

[8] J. Kovič, T. Pisanski, A. T. Balaban and P. W. Fowler, On symmetries of benzenoid systems, MATCH Commun. Math. Comput. Chem. 72 (2014), 3-26, http://match.pmf.kg.ac. rs/electronic_versions/Match72/n1/match72n1_3-26.pdf.

[9] N. J. A. Sloane (ed.), The On-Line Encyclopedia of Integer Sequences, published electronically at https://oeis.org. 


\section{Appendix A Planar regular (3, 4)-systems}

These systems may be classified by two parameters: the numbers $f_{3}$ and $f_{4}$ of triangular and square faces, as in Figure 8.

As we see, the number of these systems grows very quickly with the total number of faces $f=f_{3}+f_{4}$. There are 16 such systems with 1,2 or 3 faces.

In Figure 9 they are arranged by the increasing total number of faces. We give also their boundary faces-edges codes $c_{f, e}$ and boundary angles codes $c_{a}$. Observe that in all these cases all the faces are boundary faces.

Since for the planar regular polygonal $(3,4)$-systems all their boundary angles are multiples of $30^{\circ}$, we give the boundary angles codes also in the form of multiples of $30^{\circ}$.

The corresponding boundary faces-edges code and boundary angles codes of these $(3,4)$-systems, ordered as in Figure 9, are:

$$
\begin{array}{lll}
c_{f, e}(M) & c_{a}(M) & \\
\left(3_{3}\right) & (120,120,120)^{\circ} & =30^{\circ}(4,4,4) \\
\left(4_{4}\right) & (90,90,90,90)^{\circ} & =30^{\circ}(3,3,3,3) \\
\left(3_{2}, 3_{2}\right) & (60,120,60,120)^{\circ} & =30^{\circ}(2,4,2,4) \\
\left(4_{3}, 3_{2}\right) & (120,30,90,90,30)^{\circ} & =30^{\circ}(4,1,3,3,1) \\
\left(4_{3}, 4_{3}\right) & (90,0,90,90,0,90)^{\circ} & =30^{\circ}(3,0,3,3,0,3) \\
\left(3_{2}, 3_{1}, 3_{2}, 3_{0}\right) & (60,60,120,0,120)^{\circ} & =30^{\circ}(2,2,4,0,4) \\
\left(3_{2}, 3_{0}, 4_{3}, 3_{1}\right) & (-30,90,90,30,60,120)^{\circ} & =30^{\circ}(-1,3,3,1,2,4) \\
\left(3_{2}, 3_{1}, 4_{3}, 3_{0}\right) & (90,90,-30,120,60,30)^{\circ} & =30^{\circ}(3,3,-1,4,2,1) \\
\left(3_{2}, 4_{1}, 3_{2}, 4_{1}\right) & (120,30,30,120,30,30)^{\circ} & =30^{\circ}(4,1,1,4,1,1) \\
\left(3_{2}, 4_{0}, 3_{2}, 4_{2}\right) & (120,-30,120,30,90,30)^{\circ} & =30^{\circ}(4,-1,4,1,3,1) \\
\left(4_{3}, 4_{0}, 3_{2}, 4_{2}\right) & (90,-60,120,30,90,0,90)^{\circ} & =30^{\circ}(3,-2,4,1,3,0,3) \\
\left(4_{2}, 3_{2}, 4_{0}, 4_{3}\right) & (30,120,-60,90,90,0,90)^{\circ} & =30^{\circ}(1,4,-2,3,3,0,3) \\
\left(4_{3}, 4_{1}, 3_{2}, 4_{1}\right) & (90,0,30,120,30,0,90)^{\circ} & =30^{\circ}(3,0,1,4,1,0,3) \\
\left(4_{3}, 3_{0}, 4_{3}, 3_{1}\right) & (90,90,-60,90,90,30,30)^{\circ} & =30^{\circ}(3,3,-2,3,3,1,1) \\
\left(4_{3}, 4_{1}, 4_{3}, 4_{1}\right) & (90,90,0,0,90,90,0,0)^{\circ} & =30^{\circ}(3,3,0,0,3,3,0,0) \\
\left(4_{3}, 4_{0}, 4_{3}, 4_{2}\right) & (90,90,-90,90,90,0,90,0)^{\circ}=30^{\circ}(3,3,-3,3,3,0,3,0)
\end{array}
$$

\begin{tabular}{|c|c|c|c|c|}
\hline & $f_{4}=0$ & $f_{4}=1$ & $f_{4}=2$ & $f_{4}=3$ \\
\hline$f_{3}=0$ & & & & ! \\
\hline$f_{3}=1$ & & & & etc. \\
\hline
\end{tabular}

Figure 8: Regular planar (3,4)-systems classified by two parameters. 


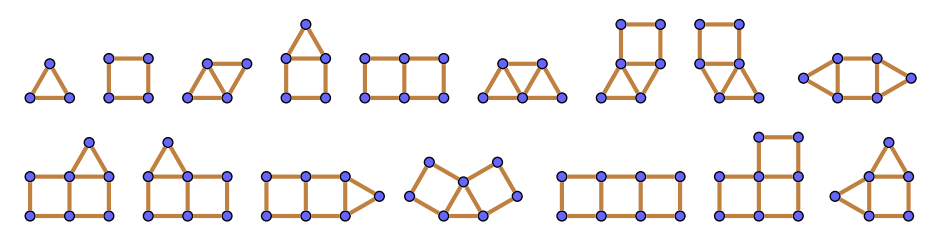

Figure 9: Planar regular polygonal $(3,4)$-systems with $f=f_{3}+f_{4} \leq 3$ faces.

\section{Appendix B Types of faces}

The idea to classify the types of hexagons in a benzenoid system with respect to their contacts to adjacent faces in a system [2,7] may be generalized to any $n$-gonal faces in any polygonal system as follows:

Definition B.1. The type of the $n$-gonal face $f$ in a polygonal system $M$ is the cyclical binary sequence $\left(b_{1}, \ldots, b_{n}\right)$ where $b_{i}=0$ if the $i$-th edge of $f$ is incident to at least one other face of $M$ and $b_{i}=1$ if it is a boundary edge of $f$. The number of types of $n$-gons having $k$ entries 1 in the binary code is denoted $T(n, k)$. The number of possible types of a $n$-gon is denoted $T(n)$.
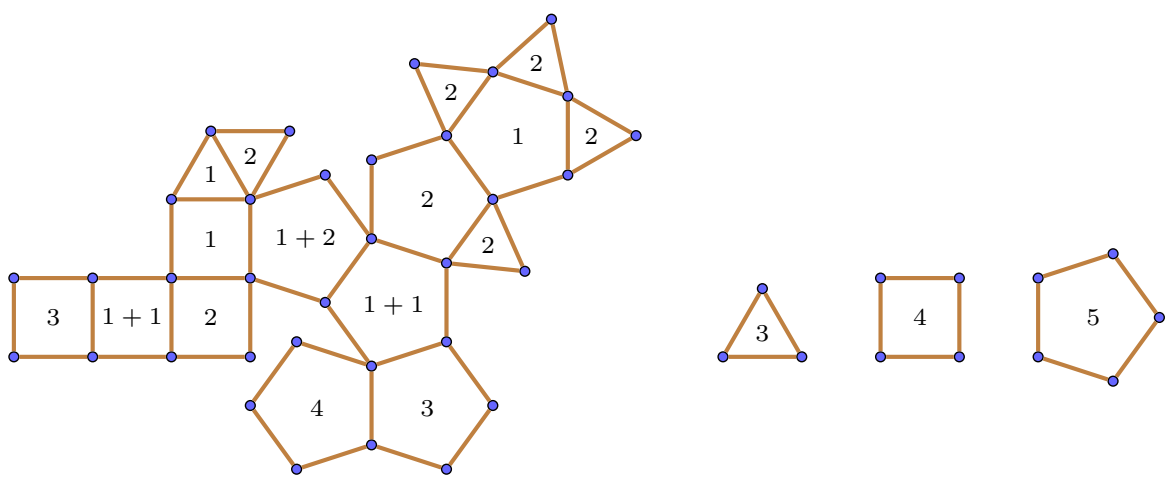

Figure 10: Boundary face types $T_{1}, T_{2}, T_{3}, S_{1}, S_{2}, S_{1+1}, S_{3}, S_{4}, P_{1}, P_{2}, P_{1+1}, P_{3}, P_{1+2}$, $P_{4}, P_{5}$.

Theorem B.2. The following relations hold:

(i) $T(n)$ equals the number of binary cyclical sequences of length $n$.

(ii) $T(3)=4, T(4)=6, T(5)=8, T(6)=13$.

Proof. (i) This is obvious, and trivially implies also relations $T(n, k)=T(n, n-k)$ and $T(n)=T(n, 0)+T(n, 1)+\cdots+T(n, n)$. To each type $t$ of a $n$-gon exists the opposite type $t^{*}$ with the entries $b_{i}^{*}=1-b_{i}$, hence $t^{* *}=t$.

For (ii) see Figure 10. Summing the adjacent entries 1 and ignoring the entries 0 we can, at least for the triangles $T$, squares $S$ and pentagons $P$, denote their possible boundary 
types like this:

$$
\begin{aligned}
& T_{0}=(0,0,0), \quad T_{1}=(1,0,0), \quad T_{2}=(1,1,0)=T_{1}^{*}, \\
& T_{3}=(1,1,1)=T_{0}^{*}, \quad S_{0}=(0,0,0,0), \quad S_{1}=(1,0,0,0), \\
& S_{2}=(1,1,0,0)=S_{2}^{*}, \quad S_{1+1}=(1,0,1,0)=S_{1+1}^{*}, \quad S_{3}=(1,1,1,0)=S_{1}^{*}, \\
& S_{4}=(1,1,1,1), \quad P_{0}=(0,0,0,0,0), \quad P_{1}=(1,0,0,0,0) \text {, } \\
& P_{2}=(1,1,0,0,0), \quad P_{1+1}=(1,0,1,0,0), \quad P_{1+2}=(1,0,1,1,0)=P_{1+2}^{*} \text {, } \\
& P_{3}=(1,1,1,0,0)=P_{2}^{*}, \quad P_{4}=(1,1,1,1,0)=P_{1}^{*}, \quad P_{5}=(1,1,1,1,1) .
\end{aligned}
$$

The inner faces are

$$
T_{0}=(0,0,0), \quad S_{0}=(0,0,0,0), \quad P_{0}=(0,0,0,0,0) .
$$

The 13 types of boundary hexagons $H$ with $1,2,3,4$ or 5 boundary edges, e.g.

$$
H_{1+1+1}=(1,0,1,0,1,0) \quad \text { or } \quad H_{2}=(1,1,0,0,0,0)
$$

are given in [2] and more precisely classified in [7].

Remark B.3. The sequence $T(n)$ (although defined only for $n \geqslant 3$ ) corresponds to the sequence A000029 in OEIS [9]. 